Voix et Images

\title{
Mise à mort de la femme et « libération " de l'homme : Godbout, Aquin, Beaulieu
}

\section{Lori Saint-Martin}

Volume 10, numéro 1, automne 1984

Littérature canadienne-anglaise

URI : https://id.erudit.org/iderudit/200460ar

DOI : https://doi.org/10.7202/200460ar

Aller au sommaire du numéro

Éditeur(s)

Université du Québec à Montréal

ISSN

0318-9201 (imprimé)

1705-933X (numérique)

Découvrir la revue

Citer cet article

Saint-Martin, L. (1984). Mise à mort de la femme et « libération » de l'homme : Godbout, Aquin, Beaulieu. Voix et Images, 10(1), 107-117.

https://doi.org/10.7202/200460ar d'utilisation que vous pouvez consulter en ligne.

https://apropos.erudit.org/fr/usagers/politique-dutilisation/ 


\title{
Mise à mort de la femme et "libération » de l'homme : Godbout, Aquin, Beaulieu
}

\author{
par Lori Saint-Martin, Université Laval
}

«Il y a dans tous mes romans un attentat contre le corps, à la fois le corps social, le corps du texte et le corps de la femme. Il est certain que, dans la fantasmatique mâle, le corps de la femme joue ce rôle de lieu privilégié pour l'attentat ${ }^{1}$ ).

Cette déclaration de principes d'Alain Robbe-Grillet est d'une pertinence certaine en ce qui concerne les œuvres de quelques écrivains québécois qui ont, eux aussi, écrit des livres-attentats. Jacques Godbout dans le Couteau sur la table, Hubert Aquin dans Trou de mémoire, Victor-Lévy Beaulieu dans Jos Connaissant mettent en scène leurs fantasmes sadoérotiques à travers les réflexions de narrateurs exploités, aliénés politiquement et socialement, enfoncés dans une existence médiocre. Pour s'en sortir, chacun passera par la même expérience «libératrice», soit la mise à mort symbolique ou réelle d'une femme. Que ce soit par la simple (?) mutilation du corps féminin (Jos Connaissant) ou par le meurtre, la femme, qui n'est évidemment pas responsable de l'exploitation économique que vit l'homme (elle en est même la première victime), sera anéantie, pour que le héros se sente libéré d'un passé et d'un présent étouffants. Pourqụoi la révolte et la libération de ces personnages doivent-elles passer par la torture d'une femme? Et pourquoi la critique ne parle-t-elle pas de ce phénomène ${ }^{2}$ ?

1. Alain Robbe-Grillet, cité par Anne-Marie Dardigna, les Châteaux d'Eros, Paris, Maspéro, 1980, p. 21.

2. L'image négative de la femme qu'on retrouve dans ces romans, la critique n'en parle jamais de façon systématique. Tout au plus dira-t-on que les femmes n'ont pas toujours le beau rôle. Voir par exemple l'article de Jeannette Urbas, «La représentation de la femme chez Godbout, Aquin et Jasmin ", dans la Revue de l'Université Laurentienne, vol. IX, no. 1, novembre 1976, pp. 103-113. 
Dans son livre sur l'éroto-pornographie française, Anne-Marie Dardigna propose une «structure érotique» qui caractérise les romans de Klossowki, de Bataille et de plusieurs autres. Il s'agit, dans ces romans, d'un discours sur la sexualité qui a pour but, entre autres, de légitimer la violence faite aux femmes. Nous nous proposons dans cet article de démontrer qu'on retrouve la même structure dans les textes québécois en question. Le thème de la sexualité dans ces romans est inséparable de la réflexion socio-politique, mystique ou autre, puisque la réflexion du personnage masculin mène justement à une rupture qui passe par la mise à mort symbolique ou réelle d'une femme.

Selon l'analyse que fait Dardigna dans les Châteaux d'Eros, cette structure érotique se résume à quatre éléments de base :

1) L'expérience des limites : le narrateur du texte fait partie d'une élite. L'érotisme sera un culte, une cérémonie souvent vécue dans l'excès. L'attirance sexuelle vers une femme c'est l'attirance vers la bestialité.

2) L'aveu : l'auteur fait avouer aux femmes que «tout est sexe en elles, et jusqu'à l'esprit ${ }^{3}$ ». Pour justifier la domination, la violence, le viol souhaités par l'homme, on prive les femmes de volonté propre, on déclare qu'elles désirent la violence. La femme ne signifiera plus que par son corps, puisqu'on lui aura interdit l'áccès à la parole.

3) La prostitution: redoutant le désir féminin signe d'autonomie, les hommes décident entre eux de l'usage qu'ils feront du corps féminin. Privées de leur individualité, neutralisées en quelque sorte, toutes les femmes deviendront, du coup, interchangeables.

4) La jouissance inégalitaire: "C'est dans la non-observation de la morale établie que se révèle et $s$ 'affirme l'élite ${ }^{4} \%$. Dans les textes érotopornographiques français, l'élite se distinguait surtout par ses richesses matérielles. Dans le roman québécois, il s'agit plutôt d'une élite intellectuelle. Mais dans les deux cas, le héros fonde sa jouissance et sa libération sur la souffrance des femmes.

Il n'est pas superflu, dans ce contexte, d'évoquer brièvement un autre lieu commun de la symbolique occidentale, soit celui qui relie la femme à la terre. Cette thématique est particulièrement frappante dans le roman québécois. L'équivalence femme/pays est trompeuse parce qu'elle traduit un glissement du particulier au général qui s'opère aux dépens de la femme. Celle-ci n'existera plus en tant qu'être humain autonome. Elle sera devenue

3. Dardigna, p. 149.

4. Dardigna, p. 207. 
le symbole d'un mythe collectif ou d'une réalité culturelle. Dans les romans dont il est question ici, le narrateur trouvera justifiée la mise à mort d'une femme parce qu'il perçoit ce meurtre en termes symboliques. Patricia (le Couteau sur la table) et Joan (Trou de mémoire) mourront parce qu'elles «symbolisent" le Canada anglais. La vraie femme disparaîtra sans que sa mort n'inquiète personne, puisqu'elle aura permis au héros de se libérer à bon compte de l'oppression politique dans laquelle la femme n'a, en réalité, presque rien à voir. Ceux qui font des femmes les responsables même symboliques de l'oppression, opèrent un renversement qu'on aura vu notamment dans les procès pour viol : ils punissent la victime pour mieux innocenter le criminel.

\section{$* * *$}

Nous n'avons pas la prétention de vouloir interpréter, à partir d'indices textuels ou biographiques, les convictions personnelles des auteurs présentés ici sur la question des femmes et de la violence. Ce que nous trouvons frappant, c'est plutôt la ressemblance des trois univers fictifs en question, à la fois entre eux et par rapport aux romans éroto-pornographiques étudiés par Dardigna. Dans tous les cas, on retrouve une vision de la femme objet et provocatrice du désir/bête obsédée par la sexualité/instrument permettant à l'hommie d'élite d'affirmer sa supériorité au moyen de la violence.

\section{Le Couteau sur la table, de Jacques Godbout}

Il est surtout question, dans ce roman, d'une relation vouée à l'échec. L'amour du narrateur et de Patricia fonctionne comme une mise en abyme des relations entre'le Canada et le Québec. Ce qui est troublant toutefois, c'est que cette allégorie politique semble justifier, au niveau de la fiction, le meurtre de Patricia par le narrateur. La révolution des hommes passe inévitablement par le corps de la femme, sans que personne n'y trouve à redire.

Comme nous tenterons de le démontrer, le Couteau sur la table reprend la structure qui caractérise les textes éroto-pornographiques étudiés par Dardigna. L'image de la femme y est sensiblement la même, quoiqu'elle soit présentée de façon moins explicite.

Il s'agit sans aucun doute, pour le narrateur et pour Patricia, d'une expérience des limites : le vagabondage à travers le pays, l'épuisement physique et moral du narrateur, les jeux et les nuits' d'amour interminables en font foi, tout comme, au niveau formel, l'ěcriture désordonnée, les phrases laissées en suspens et la rupture de l'ordre chronologique. Le narrateur s'affirme en tant que membre d'une élite intellectuelle que caractérisent l'angoisse et le doute.

Il y a polarisation des natures féminine/masculine, selon les termes de l'aveu: la femme incarne la sexualité; l'homme, l'intellect. Dès les pre- 
mières pages du roman, le narrateur nous dit qu'ils jouaient, Patricia et lui, des rôles opposés à leur réalité: "Je jouais au soldat, elle à l'intellectuelle ${ }^{5}$. Or, comme le narrateur n'est évidemment pas un soldat, Patricia n'a rien d'une intellectuelle. Elle s'enferme dans une sorte d'abrutissement sexuel, refusant de lire les journaux ou d'écouter les nouvelles (p. 26). Elle tombe de sommeil.lorsque son amant veut lui parler d'autre chose que de la vie qu'ils mènent ensemble (p. 42). Par contre, elle a une «sensualité toujours en éveil».(p. 60).

En même temps que le narrateur établit l'équivalence femme/corps, il refuse la parole féminine, non seulement celle de Patricia, la méchante Canadienne anglaise, mais aussi celle de Madeleine, la douce Québécoise. Madeleine ne parle en fait que très rarement, s'affirmant plutôt comme une présence silencieuse. Les propos prêtés à Patricia sont d'une bêtise et d'une mauvaise foi stupéfiantes. Citons à titre d'exemple: «Je ne suis pas une raciste», dit-elle, «mais les seuls nègres que $\mathrm{j}$ 'ai connus étaient porteurs à bord des trains» (p. 29). L'aveu est donc celui de l'incapacité féminine à prendre la parole. Réduite au silence, rejetée hors du discours, la femme n'est plus qu'un «mannequin» (p. 36), elle n'a plus que son corps.

Comme dans les romans éroto-pornographiques frảnçais, le narrateur décrira la sexualité féminine en des termes imprégnés de mépris. La femme aimée ressemblera à s'y méprendre à une prostituée: Patricia se fait "garce» lorsque les hommes la regardent (p. 78). Elle «faisait le campus comme les putains font la rue Saint-Denis » (p. 53), ce qui en dịt long sur cette femme qui jouait à l'intellectuelle.

Pour se décoloniser, le narrateur doit rejeter tout lien avec le monde anglophone. Dans l'univers fictif du Couteau sur la table, c'est la femme qui symbolise l'oppression et c'est uniquement par la souffrance des femmes que le narrateur pourra s'affirmer.

Dans un premier temps, on prive la femme de son identité propre en la caractérisant selon les termes de la dichotomie chrétienne traditionnelle. Patricia fait penser à Eve «avec ses seins comme des pommes sous un pull pour Adam " (p. 129). Madeleine ressemble plutôt à la Vierge douce, belle, soumise et, surtout, féconde.

Les deux femmes seront d'ailleurs réduites à une autre image traditionnelle, celle de la femme/terre/territoire, dont nous avons déjà mis en valeur le tort qu'elle fait aux femmes : «Patricia n'était plus qu'un territoịre abandonné au premier Anglais venu, Madeleine, elle, était ce pays conquis que je retrouvais lentement, calmement». (p. 105).

5. Jacques Godbout, le Couteau sur la table, Paris, Senil, 1965, p. 18. 
Dans un deuxième temps, on procède à la mise à mort de la femme. Madeleine sera la victime d'un accident de moto et on n'entendra plus parler d'elle. Le narrateur prendra «le couteau sur la table» pour se débarrasser de Patricia.

Tout au long du récit, le narrateur affirme être fasciné par la société du «clinquant» et du. «bisenesse» à laquelle appartient Patricia (pp. 27-28). Tuer Patricia, pour lui, signifierait tuer en lui-même cette fascination et, du coup, la dépendance coloniale, comme l'indique la présence à la toute fin du roman de l'article de journal portant sur les activités du FLQ. Le meurtre lui permettra de s'affirmer en tant que Québécois voué à la révolution. Il sait pourtant que Patricia n'est que «la fille d'un ennemi» (p. 43) et non une ennemie. Le capital étranger, le contrôle des matières premières et de l'industrie par des compagnies non québécoises; les hommes pólitiques canadiens qui votent des lois permettant de maintenir le Québec en état d'infériorité politico-économique: voilà les responsables de la «prolétarisation » du peuple québécois ${ }^{6}$. On voit mal ce que la mort d'une femme, aussi riche soit-elle, pourra y changer.

Débarrassé enfin de Madeleine et de Patricia, le narrateur aura tué, plutôt que les vestiges de colonisé en lui-même, toute dépendance vis-à-vis des femmes. La fin du livre dit moins une libération politique que l'anéantissement de la. femme.

\section{Trou de mémoìrë, d'Hubert Aquin}

Dans ce roman, le corps social, le corps du texte et le corps de la femme dont parlait Robbe-Grillet n'en font plus qu'un, manipulé au gré du narrateur «révolutionnaire». 11 y a refus de l'ordre social, rèfus de l'écriture traditionnelle, refus àussi de la femme que l'auteur réduira d'abord au statut de symbole pour ensuite faire d'elle un instrument permettant à l'homme de déclencher la révólution.

Il n'est pas surprenant alors que la structure érotique décrite par Dardigna se retrouve intacte dans Trou de mémoire.

Le livre raconte une expérience des limites dans tous les sens du mot. Grâce aux drogues et à l'ivresse qui suit le meurtre, le narrateur s'affirmera unique: «Je suis présent d'une présence réelle, jè suis comme jamais un homme n'a été $\left.{ }^{\text {}}\right)$. Il se déclare en même temps fondateur d'une politique révolutionnaire: «(Il faut) appeler la révolution avant de la faire. L'écrire minutieusement... (...) Personne n'écrit sauf moi» (pp. 55, 57).

6. Jean Hamelin, l'Histoire du Québec, Montréal, France/Amérique, 1976, p. 498. Voir aussi entre autres p. 494, 502.

7. Hubert Aquin, Trou de mémoire, Ottawa, Le cercle du livre de France, 1968, p. 20. 
Comme dans les romans éroto-pornographiques; le narrateur éprouvera le besoin de se voir comme un être tout-puissant à qui obéissent; fascinées, les femmes: «... je n'arrêtais pas de faire travailler Joan (...) selon mon plan et mon caprice (...) elle se trouvait aux mains d'un maître immoral, moi, et elle avait peur " (pp. 67-68).

L'homme d'élite se distingue entre autres par le fait que, poụr lui, toutes les femmes sont interchangeables. Il dit préférer à la triste répétition conjugale des rencontres-éclairs anonymes:

...je m'imagine en train de relever la robe d'une collégienne ou d'une inconnue qui ne veut pas plus me connaître que je ne veux l'inscrire sur mon carnet d'adresses - mais qui serait prête à cela, pourvu que tout se passe rapidement, violemment, sans le moindre conditionnement sentimental ou social (p. 116)...

Magnant refuse donc tout engagement, fût-il momentané. De plus, la jouissance du personnage se fonde explicitement sur le malheur des femmes. Magnant nous le dit en des termes assez clairs: «Faire l'amour normalement ne m'intéresse plus vraiment (...) Je viole, chaque nuit, un nombre toujours croissant de belles inconnues que j'abandonne terrifiées i) $(p, 112$, 117).

Peu importe d'ailleurs que dans l'exemple que nous venons de citer, il s'agisse des fantasmes du narrateur; le roman n'est-il pas savamment construit pour qu'on ne puisse distinguer le «réel» de l'imaginé? De toute façon, le roman est fatalement le produit d'un fantasme d'auteur. La pornographie est dangereuse pour les femmes justement parce qu'elle met en scène des fantasmes masculins qu'on présente comme étant bien réels, tel le prétendu masochisme féminin qui ferait que toutes les femmes désirent être violées, battues, violentées ${ }^{8}$. Le même phénomène se retrouve chez Aquin : au désir masculin de violer répond fatalement, chez la femme, le désir d'être violée. Suivant cette logique, $R R$ aurait demandé à Magnant de la violentẹ :

Pierre-Xavier, disait-elle, fais-moi jouị moi aussi, ne sois pas cruel. Je veux que tu me fasses comme à Joan (p. 182) ...

L'aveu dont parlait Dardigna se retrouve ici sous sa forme la plus pure. La violence, le viol sont déclarés jouissifs pour la femme, selon une «logique» étroitement tautologique :

... bien sûr, RR est une femme et, sans doute, : a-t-elle joui d’être pénétrée par un homme - et cela, même 'si c'était sous contrainte (p. 171).

L'auteur rejettera sur la femme l'animalité du viol: Olympe imagine RR «faisant l'amour comme une bête» avec Magnant (p. 189).

8. Voir entre autres le livre de Dardigna. 
Bien plus, le viol devient dans Trou de mémoire l'expérience qui transforme de façon positive la vie d'une femme. RR nous dira qu'elle n'avait «jamais eu le sentiment d'être une femme normale» avant de connaître Magnant (p. 125). Maintenant, tout est différent : «... je suis une autre femme: heureuse, détendue, nouvelle» (p. 203). Elle changera de nom et d'identité, devenant une Québécoise pure laine. Dans un renversement spectaculaire, RR a appris à aimer son agresseur :

...j'en suis venue à aimer celui qui, s'ennuyant follement de Joan, est venu jusqu'à Lagos pour en retrouver l'image (p. 203)...

Le meurtrier lui fait pitié maintenant, et non plus la victime.

Par le discours idéologique et par les événements romanesques qui y sont présentés, Trou de mémoire est, entre autres, une apologie de la violence faite aux femmes. Faire dire à la victime, par le truchement d'un faux «je» féminin, qu'elle a joui du viol et qu'elle aime son violeur, revient à déclarer que le viol n'existe pas, que le meurtre ne fait pas mal. Joan est morte "égorgée de plaisir» (p. 26, c'est nous qui soulignons) et les critiques sont d'accord avec RR que c'est plutôt ce pauvre Magnant, violeur et meurtrier, qui fait pitié, puisque Joan, morte, ne cesse de le hanter ${ }^{9}$. Par contre, l'agression dirigée contre l'homme est condamnée par l'auteur, comme dans le cas d'Olympe qui doit passer une nuit en prison à Genève à cause de la police raciste. Il n'est jamais question de suggérer que l'expérience a été jouissive pour lui. Le viol et le meurtre se trouvent donc innocentés, légitimés, pourvu que la victime en soit une femme. Cette "logique» implacable prive la femme du droit de refuser la violence qui s'exerce contre elle. Sans ce droit fondamental, la femme n'a plus aucune autonomie, elle existe à peine. Elle ne sera plus que ce que l'homme qui la désire veut bien faire d'elle.

Au centre de la philosophie de P.-X. Magnant, se retrouve le lieu qu'il établit entre la puissance sexuelle et la puissance politique :

Mon comportement sexuel est à l'image d'un comportement national frappé d'impuissance; plus ça va, plus je sens bien que je veux violer (p. 112)...

9. René Lapierre écrit, dans l'Imaginaire captif: Hubert Aquin, Montréal, Quinze, 1981, que chez Aquin, le viol et le meurtre «sacralisent les « victimes" et se retournent contre les narrateurs» (p. 73). C'est donc du meurtrier qu'il faudrait avoir pitié et ce serait la victime (à noter que Lapierre met le mot "victime» entre guillemets pour en minimiser la portée) qui, en fin de compte, l'emporterait. Il s'agit là d'une bien triste «victoire" que la femme ne pourra acheter que par sa propre mort! 
Or, une fois établie cette équivalence : impuissance sexuelle = incapacité d'agir, tous les moyens de s'en sortir se voient, du coup, justifiés. Comme le disait si clairement Robbe-Grillet, «le corps de la femme joue ce rôle de lieu privilégié pour l'attentat 10 m. Ici comme dans le Couteau sur la table, le narrateur fait d'une riche Canadienne anglaise la responsable de l'oppression du Québec. Tuer cette femme deviendra dès lors «un acte révolutionnaire ${ }^{11} »$ qui fera du meurtrier un héros national:

... en tuant Joan, $\mathrm{j}$ 'ai engendré l'histoire d'un peuple sevré de combats et presque mort de peur à force d'éviter la violence (p. 87).

Les critiques littéraires trouvent tout à fait légitime qu'une femme meure pour mettre fin à l'oppression politique établie par les hommes de son peuple. Selon l'expression de Gilles de la Fontaine,

... pour Aquin, la seule vérité du peuple québécois reste à trouver audelà $(. .$.$) du «crime parfait » commis envers son double anglophone$ dans son identité à reconnaître et à affirmer ${ }^{12}$.

Personne ne trouve étrange que l'identité des hommes s'affirme aux dépens des femmes.

"Tout se passe sous le signe du blasphème et de l'action », écrit Magnant (p. 57). «Nous sommes frères par le sang que nous ferons verser un jour...» (p. 35). Cette fraternité exclut évidemment les femmes. Celles-ci signifieront désormais un ordre social à refuser. «Joan est le symbole de tous les pays où règne l'oppression ${ }^{13}$ ", selon l'expression d'un critique. La femme-symbole passera vite à une autre étape de la réification : elle deviendra l'instrument qui permet à l'homme de déclencher la révolution.

Texte qui se veut révolutionnaire, Trou de mémoire ne véhicule, au sujet des femmes, que les idées reçues les plus courantes de la pornographie. Privée de volonté propre, victime consentante de la violence masculine, la femme aquinienne paie de sa vie la libération de l'homme.

\section{Jos Connaissant, de Victor-Lévy Beaulieu}

Le but avoué de Jos, c'est de devenir un saint 14. Pour ce faire, il doit se purifier, mais il y a un obstacle: Marie, la serveuse, court après lui et il la désire tout en la méprisant. Il faudra tuer en lui-même le besoin qu'il a de la femme, ce qu'il fait en rejetant sur celle-ci tout le poids de la sexualité afin de devenir, lui, tout esprit.

10. Dardigna, p. 21.

11. M. E. Kidd, "La thématique de l'eau dans l'œuvre d'Hubert Aquin », dans Voix et Images, vol. IV, no. 2, décembre 1978, p. 265.

12. Gilles de la Fontaine, Hubert Aquin et le Québec, Montréal, les Éd. Parti Pris, 1977, p. 70.

13. M. E. Kidd, p. 265.

14. Victor-Lévy Beaulieu, Jos Connaissant, Montréal, VLB éditeur, 1978, p. 172. 
Jos vis une expérience des limites: limites du désespoir (la mort de Mam) et limites de la bestialité (la semaine qu'il passe enfermé avec Marie). Avec quelques autres âmes tourmentées (Abel, Malcomn) il appartient à une élite qui se distingue des autres par son mysticisme: «... je passe toute la nuit éveillé parce qu'il faut qu'il y ait quelqu'un qui songe à l'Apocalypse" (p. 14).

L'aveu attribué à la femme est double. Premièrement, elle sera incapable de prendre la parole ou de réfléchir à l'avenir du monde. Marie avoue à plusieurs reprises qu'elle ne comprend pas Jos, qu'il lit des livres trop difficiles pour elle, que les discours de son amant la dépassent (pp. 58, 62, 166). L'homme impose sa domination au nom de l'esprit et de l'intelligence, qualités dont la femme semble dépourvue.

Deuxièmement, le narrateur charge la femme de tout le poids de la sexualité qu'il voit comme étant sale, laide, bestiale. Jos croit que Marie est peut-être "un vampire ou quelque animal fabuleux" (p. 184). La "bête abjecte de son sexe» (p. 186) fait peur. Marie est sale et dégoûtante : comment oublier l'image de cette femme «en train de vomir sur les draps (...) les cuisses pleines de détritus qui sortaient d'elle» (p. 185). Après avoir battu Marie, Jos la décrit en termes de pourriture: "zmarie lépreuse, Marie décomposée déjà et honteuse sous le voile noir derrière lequel sa face était une plaie purulente» (p. 231).

Pour ce qui est de l'interchangeabilité des femmes, Jos Connaissant va encore plus loin que les autres livres présentés ici. Au lieu de coucher avec toutes indifféremment, Jos refuse d'emblée toutes les femmes:

Ce vieux mythe de l'amour. Est-ce que ça vaut la peine de vivre cent ans pour courir après des femmes et sucer des vulves et faire des petits morveux qui deviendront de grosses têtes carrées et (p. 13)...

Jos veut atteindre à la perfection par la voie du dépouillement. Il mène une vie pure : «Je vis seul, et je travaille, et je prie» (p. 92). Seuls ses désirs sexuels l'empêchent de devenir un saint. Il s'en libérera donc en détruisant Marie qui, pour lui, incarnait la sexualité.

Dès le début, Jos dit qu'il déteste Marie. Il désire quand même la violer (p. 13). Il méprise Marie parce qu'elle a déjà été danseuse et parce qu'elle ne le comprend pas. Cette femme en vient à représenter pour lui le néant, la mort : elle est stérile et frigide depuis son cancer ( $p p .165,192$ ). La semaine que Jos passe avec elle «ne (lui) avait appris que le grand besoin qu'(il) avai $(\mathrm{t})$ de ( $\mathrm{sa}$ ) solitude et de ('son) mépris » ( $\mathrm{p}$. 192). Il battra Marie à deux reprises, mais elle refusera de porter plainte. «Je t'en veux pas, Jos» dira-telle (p. 229). Elle acceptera sans comprendre la violence dirigée contre elle.

Jos fait de Marie l'instrument de son rejet de la sexualité, comme il l'affirme lui-même :

... aller pourtant jusqu'au bout et entrer en elle avec violence (...) et la briser de partout et la détruire (...) - c'était la seule façon que j'avais 
de la conserver, de la tuer et de me l'approprier, elle fétiche, elle corps initiatique grâce auquel j'allais me vaincre et m'aimer» (p. 181. C'est nous qui soulignons. ).

Dans ce livre, toutes les femmes sont rejetées d'emblée puisqu'elles n'existent que par leur corps puant et voué à la mort. Seule la mère sera sauvée, puisqu'il y a chez elle la pureté refusée aux autres: «... douze enfants n'ont pas atteint la virginité de Mam ", dira Jos (p. 113).

Seuls les quelques initiés ont accès à la pureté mystique. L'intelligence, la réflexion, le discours sont interdits aux femmes. La mise à mort symbolique de la femme équivaut à une condamnation sans appel. Jos Connaissant va bien plus loin que le Couteau sur la table et Trou de mémoire. La femme représente ici la bêtise, la bestialité, la mort. En se libérant d'elle, l'homme commence la conquête d'un monde meilleur: "J'allais devenir l'image de ce pays (...) je ne pourrais rien imaginer de trop fou (...) car c'était au fond du délire que ce pays se reconnaîtrait et s'assumerait " (p. 265). Ce livre dit non seulement la mort de la mère, mais aussi, à travers le rejet de Marie, la mise à mort du besoin de la femme chez l'homme.

Nous avons vu que les romans étudiés ici reprennent très exactement le discours sur la femme que vẻhiculent les romans éroto-pornographiques français. Privée de la parole, la femme n'est plus qu'un corps, qu'une bête, qu'un instrument qui permet à l'homme d'élite de s'affirmer par-la transgression de l'ordre établi.

Cette vision de la femme est d'autant plus nuisible qu'elle ne s'exprime pas toujours, dans les textes québécois, aussi clairement que chez un RobbeGrillet. La problématique nationaliste ou mystique y occupe l'avant-scène; la conception de la femme en découle et y est subordonnée. Serait-ce pour cette raison que l'idéologie que véhiculent ces romans au sujet de la femme n'a jamais été étudiée?

Les romans que nous avons analysés ici ne sont pas l'œuvre d'écrivains. inconnus ou sans importance. On a dit de Godbout qu'il figure «parmi les écrivains de langue française les plus significatifs de notre époque ${ }^{15}$ 》, d'Aquin qu'il a fait «la première ouvre géniale de notre littérature ${ }^{16}$, Quant à Victor-Lévy Beaulieu, même ceux qui n'aiment pas ses romans reconnaissent chez lui une écriture significative et nouvelle. On fait l'éloge de ces romans, on les donne à lire aux étudiants, ils font le sujet de beaucoup de thèses et d'articles, sans qu'on mentionne jamais l'idéologie réac-. tionnaire qu'ils véhiculent au sujet de la femme. N'est-ce pas cautionner, de façon implicite, le sexisme?

15. Étienne Lalou, citation reproduite sur la couverture de Salut Galarneau, Paris, Seuil, 1967.

16. Réginald Martel, dans la Presse, 19 mars 1977. 
Niant la réalité de l'oppression politico-économique, ces romans font de la femme la responsable de cet état de choses. Au Canada et au Québec, pourtant, les femmes sont presque totalement absentes de ces lieux de pouvoir que sont le gouvernement, l'industrie et la finance. En 1983, «on compte seulement $5 \%$ de femmes dans des métiers techniques demandant une qualification particulière, et $5 \%$ de femmes à des postes de direction ou de responsabilité ${ }^{17}$ '). Il n'y a que treize femmes députées à la Chambre des communes et sept à l'Assemblée nationale du Québec ${ }^{18}$. Comment peut-on alors rendre ces êtres sans pouvoir responsables de la colonisation du Québec? Tuer Patricia et Joan pour leur faire payer l'oppression du peuple québécois, c'est, encore une fois, punir les victimes. Une révolution qui ne s'attaque pas à la racine du mal a-t-elle des chances de réussir?

Les romans en question présentent des narrateurs qui tentent de légitimer la domination de la femme par l'homme en évoquant soit l'oppression politique qu'ils subissent, soit leurs tâtonnements phycho-mystiques. Lorsqu'on prend conscience que ces romans de soi-disant libération personnelle et nationale véhiculent, au sujet de la femme, la même idéologie que la pornographie, il faut bien se rendre compte à quel point notre littérature est truquée. Lieu de la mise à mort de la femme, comment peut-elle dire l'épanouissement du peuple québécois?

Réduire la femme à ne plus être qu'un symbole, c'est nier qu'elle existe en tant qu'être humain autonome. Trouver légitimes le viol et le meurtre qui font déclencher l'écriture ou la révolution, c'est dire que la libération de l'homme est sans prix et la liberté, la vie même, de la femme, sans valeur.

17. Collectif, Terre des femmes, Paris/Montréal, La Découverte/Maspero/Boréal Express, 1983, p. 202.

18. Lysiane Gagnon, Vivre avec les hommes, Montréal, Québec/Amérique, 1983, p. 162. 